\title{
Lengths and volumes in Riemannian manifolds
}

\author{
Christopher B. Croke* \\ Nurlan S. Dairbekov ${ }^{\dagger}$
}

December 31, 2002

\begin{abstract}
We consider the question of when an inequality between lengths of "corresponding" geodesics implies a corresponding inequality between volumes. We prove this in a number of cases for compact manifolds with and without boundary. In particular, we show that for two Riemannian metrics of negative curvature on a compact surface without boundary, an inequality between the marked length spectra implies the same inequality between the areas with equality implying isometry.
\end{abstract}

\section{Introduction}

In this paper we consider the question of inequalities on volumes implied by inequalities on lengths of geodesics. This is in fact a collection of related problems.

The problem for a $(M, \partial M)$ a compact manifold $M$ with boundary $\partial M$ would be to compare the volumes, $\operatorname{Vol}\left(g_{0}\right)$ and $\operatorname{Vol}\left(g_{1}\right)$ (in two dimensions we use $A\left(g_{0}\right)$ and $A\left(g_{1}\right)$ ), of two Riemannian metrics $g_{0}$ and $g_{1}$ on $M$ if we know that for every pair of points $x, y \in \partial M$ we have $d_{g_{0}}(x, y) \leq d_{g_{1}}(x, y)$. In the above $d_{g_{i}}(x, y)$ represents the distance in $\mathrm{M}$ with respect to the the metric $g_{i}$, i.e. the length of the $g_{i}$-shortest path in $M$. We sometimes call $d_{g_{i}}(x, y)$ the chordal distance between $x$ and $y$. Of course, without some further assumptions on the metrics (such as some minimizing property of geodesics) there is no general comparison between the volumes.

The corresponding question for compact manifolds $N$ without boundary involves the marked length spectrum. The marked length spectrum for a Riemannian metric $g$ on $N$ is a function, $M L S_{g}: \mathcal{C} \rightarrow \mathbb{R}^{+}$, from the set $\mathcal{C}$ of free homotopy classes of the fundamental group $\pi_{1}(N)$ to the nonnegative reals. For each $\langle\gamma\rangle \in \mathcal{C}, M L S_{g}(\langle\gamma\rangle)$ is the length of the shortest curve in $\langle\gamma\rangle$ (always a geodesic). We consider two Riemannian metrics $g_{0}$ and $g_{1}$ on $N$ such that $M L S_{g_{1}}(\langle\gamma\rangle) \geq M L S_{g_{0}}(\langle\gamma\rangle)$ for all free homotopy classes $\langle\gamma\rangle$ (we then say $\left.M L S_{g_{1}} \geq M L S_{g_{0}}\right)$ and ask if $\operatorname{Vol}\left(g_{1}\right)$ must be greater than or equal to $\operatorname{Vol}\left(g_{0}\right)$. Again this is hopeless without further assumptions.

*Supported by NSF grants DMS 99-71749 and DMS 02-02536 and CRDF Grant RM2-2242.

${ }^{\dagger}$ Supported by CRDF Grant RM2-2242 and RAN. 
The natural setting in the manifold without boundary case is in negative curvature. Here there are lots of closed geodesics but exactly one for each free homotopy class (achieving the minimum length in that class). It was conjectured in [C-D-S] that for two negatively curved metrics, $g_{0}$ and $g_{1}$, on a manifold $N$ the inequality $M L S_{g_{1}} \geq M L S_{g_{0}}$ would imply $\operatorname{Vol}\left(g_{1}\right) \geq \operatorname{Vol}\left(g_{0}\right)$. It was further conjectured that equality would hold if and only if $g_{0}$ and $g_{1}$ are isometric. Our most general result proves this for surfaces (two dimensional manifolds):

Theorem 1.1. If $g_{0}$ and $g_{1}$ are metrics of negative curvature on a surface $N^{2}$ such that $M L S_{g_{1}} \geq M L S_{g_{0}}$, then $A\left(g_{1}\right) \geq A\left(g_{0}\right)$. Further, if $A\left(g_{1}\right)=A\left(g_{0}\right)$ then $g_{1}$ is isometric to $g_{0}$.

The relation between length and volume is more natural when the metrics are pointwise conformal. The higher dimensional version of the above holds in this case.

Theorem 1.2. Let $g_{0}$ be a metric of negative curvature on $N^{n}$ and let $g_{1}=$ $f^{2}(x) g_{0}$ for some smooth positive function $f$ on $N$. Then if $M L S_{g_{1}} \geq M L S_{g_{0}}$, then $\operatorname{Vol}\left(g_{1}\right) \geq \operatorname{Vol}\left(g_{0}\right)$. Further, if $\operatorname{Vol}\left(g_{1}\right)=\operatorname{Vol}\left(g_{0}\right)$ then $f \equiv 1$.

We now turn to the case of manifolds with boundary. The problem first showed up in consideration of the boundary rigidity problem. In this problem one considers the case when $d_{g_{0}}(x, y)=d_{g_{1}}(x, y)$ for all boundary points $x$ and $y$ and asks if $g_{0}$ must be isometric to $g_{1}$. Again this cannot hold in general. A survey of what is known about this problem can be found in [Cr3]. There are a few natural choices for assumptions in this case. The most general natural such assumption is $S G M$. The SGM condition (which is given in terms of $d_{g}: \partial M \times \partial M \rightarrow \mathbb{R}$ only) is harder to define. A precise definition is given in [Cr1], but loosely speaking it means that all nongrazing geodesic segments are strongly minimizing. By a nongrazing geodesic segment we will mean a segment of a geodesic which lies in the interior of $\mathrm{M}$ except possibly for the endpoints. A segment is said to minimize if its length is the distance between the endpoints and to strongly minimize if it is the unique such path. Of course this loose definition seems to rely on more than $d_{g}$ but the relationship is worked out in [Cr1]. Examples of such $(M, \partial M, g)$ are given by compact subdomains of an open ball, B, in a Riemannian manifold where all geodesics segments in B minimize. The only reason not to use the "loose" definition above is that using a definition in terms only of $d_{g}$ guarantees that if $d_{0}(x, y)=d_{1}(x, y)$ and $g_{0}$ is SGM then $g_{1}$ will be as well. In fact, all theorems stated here for the SGM case also hold for manifolds satisfying the loose definition, so the reader can treat that as a definition of $S G M$ for the purpose of this paper.

The most general result one could hope for would be of the form: If $g_{0}$ is an SGM metric on $(M, \partial M)$ and $g_{1}$ is another metric with $d_{g_{0}}(x, y) \leq d_{g_{1}}(x, y)$ then $\operatorname{Vol}\left(g_{0}\right) \leq \operatorname{Vol}\left(g_{1}\right)$ with equality of volumes implying isometry of the metrics. This is still very much an open question, which as stated includes the boundary rigidity problem. In this paper we prove a two dimensional version and prove the higher dimensional version for conformal metrics. 
The proof of the conformal case is essentially given in [Cr2], however as the version in [Cr2] (which was concerned only with boundary rigidity) makes stronger assumptions and since the proof is short we include a slightly different version of it here.

Theorem 1.3. Let $\left(M, \partial M, g_{0}\right)$ be an $S G M$ manifold with boundary and let $g_{1}=f^{2} g_{0}$ be such that $d_{g_{1}}(x, y) \geq d_{g_{0}}(x, y)$ for all $x, y \in \partial M$. Then $\operatorname{Vol}\left(g_{1}\right) \geq$ $\operatorname{Vol}\left(g_{0}\right)$. Further, if $\operatorname{Vol}\left(g_{1}\right)=\operatorname{Vol}\left(g_{0}\right)$ then $f \equiv 1$

We now consider the case where $g_{0}$ and $g_{1}$ are not assumed to be conformal. We digress for a little to discuss the higher dimensional case even though we will contribute a theorem only in two dimensions. In [Gr1] Gromov introduced the notion of Filling Volume $\operatorname{FillV} \operatorname{Vol}\left(N^{n}, d\right)$ for a compact manifold $N$ with a metric $d$ (here $d$ is a distance function which is not necessarily Riemannian). For the actual definition one should see [Gr1], but it is shown in [Gr1] that when $n \geq 2$

$$
\text { FillVol }\left(N^{n}, d\right)=\inf _{g} \operatorname{Vol}\left(M^{n+1}, g\right)
$$

where $M$ is any fixed manifold such that $\partial M=N$ (one can even take $M=$ $N \times[0, \infty)$ ), the infimum is taken over all Riemannian metrics $g$ on $M$ for which the boundary distance function is $\geq d$. In the case where $d$ is the chordal distance function of some Riemannian $\left(M^{n+1}, \partial M=N, g_{0}\right)$ one can not only fix the topology of $M^{n+1}$ but also restrict to metrics $g$ which agree with $g_{0}$ when restricted to $N$ (see [Cr3]). The filling volume is not known for many $(N, d)$, but a positive answer to our question would say that FillVol $(\partial M, d)=\operatorname{Vol}(M)$ for an SGM manifold $(M, \partial M, g)$, where $d$ is the chordal distance and further that the minimal filling is unique. Gromov in [Gr1] proved this for $M^{n}$ a compact subdomain of $\mathbb{R}^{n}$. The minimal entropy theorem of Besson, Courtois, and Gallot [B-C-G2, B-C-G1] can be used to prove the result for compact subdomains of symmetric spaces of negative curvature (see [Cr3]). For general convex simply connected manifolds $\left(M, \partial M, g_{0}\right)$ of negative curvature there is a $C^{3}$ neighborhood in the space of metrics such that any metric $g_{1}$ in that neighborhood with $\left.g_{1}\right|_{\partial M}=\left.g_{0}\right|_{\partial M}$ and $d_{g_{1}}(x, y) \geq d_{g_{0}}(x, y)$ has $\operatorname{Vol}\left(g_{1}\right) \geq \operatorname{Vol}\left(g_{0}\right)$ and equality of the volumes implies $g_{1}$ is isometric to $g_{0}$ (see [C-D-S]). This leads to the conjecture that for any compact subdomain of a simply connected space of negative curvature of dimension $\geq 3$ the filling volume of the boundary with the chordal metric is just the volume of the domain and that the domain is the unique volume minimizing filling. Of course this conjecture is still open.

We now turn to the two dimensional case. Recently Ivanov [Iv] considered the case of compact metrics $g_{0}$ and $g_{1}$ on a disk. He assumes that $g_{0}$ is a convex metric in the sense that every pair of interior points can be joined by a unique geodesic. He proves that if $d_{g_{1}} \geq d_{g_{0}}$ then $A\left(g_{1}\right) \geq A\left(g_{0}\right)$. He also says that equality in the area would imply that $d_{g_{1}}=d_{g_{0}}$. Of course, going from here to isometry of $g_{0}$ and $g_{1}$ is the boundary rigidity problem. This boundary rigidity problem is solved [Cr2, Ot2] in the case that the metric $g_{0}$ has negative curvature. Using completely different methods, we prove a similar though somewhat different result.

Since we will not assume that our surfaces are simply connected we will need to worry about the homotopy class of a path between two boundary points. So 
for boundary points $x$ and $y$, we let $\pi_{1}(x, y)$ represent the homotopy class of curves in $M$ between $x$ and $y$ and let

$$
\mathcal{A}=\left\{(x, y,[\alpha]) \mid x, y \in \partial M \text { and }[\alpha] \in \pi_{1}(x, y)\right\} .
$$

Of course, in the case of a disk $\mathcal{A}$ is just $\partial M \times \partial M$. We will define $L_{g}(x, y,[\alpha])$ to be the length of the shortest curve from $x$ to $y$ in $[\alpha]$.

There is a natural measure on the space $\Gamma_{g}$ of nongrazing $g$-geodesics between boundary points, and a map $F: \Gamma_{g} \rightarrow \mathcal{A}$ defined by $F(\gamma)=(\gamma(0), \gamma(L),[\gamma])$ where $L$ is the $g$-length of $\gamma$. We get a measure $\mu_{g}$ on $\mathcal{A}$ by pushing the natural measure on $\Gamma_{g}$ forward via $F$, and define $f_{g}: \mathcal{A} \rightarrow \mathbb{R}^{+}$by $\mu_{g}=f_{g}(x, y,[\alpha]) d x d y$ where $d x$ and $d y$ represent the arclength along the boundary. Note that, unless $(M, \partial M, g)$ is a convex metric on a disk, $f_{g}(x, y,[\alpha])$ will be 0 for many values of $(x, y,[\alpha])$; namely those for which there is no nongrazing geodesic segment in $[\alpha]$ from $x$ to $y$. (In fact, when $M$ is $S G M$ for each $(x, y) \in \partial M \times \partial M$ there is at most one $[\alpha]$ for which $f_{g}(x, y,[\alpha]) \neq 0$.) On the other hand it is not hard to see that $f_{g}$ will be smooth at points $(x, y,[\alpha])$ where there is such a geodesic segment and the geodesic segment is not tangent to the boundary at either endpoint. The fact that $\int_{\mathcal{A}} L_{g}(x, y,[\alpha]) \mu_{g}=2 \pi A(g)$ is a consequence of Santaló's formula (see section 3 ). However, somewhat surprisingly, there is a formula for the difference of the areas of two $S G M$ metrics on a surface:

Theorem 1.4. Let $_{g_{0}}$ and $g_{1}$ be two SGM metrics on a compact two-dimensional surface $(M, \partial M)$. Then

$$
A\left(g_{1}\right)-A\left(g_{0}\right)=\frac{1}{2 \pi} \int_{\mathcal{A}}\left[L_{g_{1}}(x, y,[\alpha])-L_{g_{0}}(x, y,[\alpha])\right]\left[\mu_{g_{1}}+\mu_{g_{0}}\right] .
$$

Of course the content of the proof is that the cross terms cancel. A nearly immediate consequence of this result, which relates lengths to areas, is:

Corollary 1.5. Let $g_{0}$ and $g_{1}$ be two SGM metrics on a compact two-dimensional surface $(M, \partial M)$ such that $d_{g_{1}} \geq d_{g_{0}}$. If further the $g_{1}$-length of each nongrazing $g_{1}$-geodesic $\gamma$ joining boundary points is greater than or equal to the minimal $g_{0}$ length of paths homotopic to $\gamma$ with fixed endpoints, then $A\left(g_{1}\right) \geq A\left(g_{0}\right)$; moreover, if these areas coincide then $d_{g_{1}}(x, y)=d_{g_{0}}(x, y)$ for all pairs of boundary points $(x, y)$ such that there is either a $g_{0}$ or a $g_{1}$ nongrazing geodesic between them.

In particular, if $(M, \partial M)$ is topologically a disk and if $d_{g_{1}} \geq d_{g_{0}}$ then $A\left(g_{1}\right) \geq A\left(g_{0}\right)$.

We would like to thank Philippe Thieullen for providing us with preprint [L-T] and for useful conversations.

\section{Manifolds without boundary}

The main tools used in the proof of Theorem 1.1 are Bonahon's intersection of geodesic currents $[\mathrm{Bo}]$ and Sigmund's theorem [Si] about the denseness of measures supported on closed orbits of an Axiom A flow (in particular the geodesic 
flow of a negatively curved metric) in the space of flow invariant probability measures.

We first review the notion of geodesic current in our setting. Let $\left(N^{2}, g\right)$ be a compact surface of negative curvature and $(\tilde{N}, \tilde{g})$ its universal covering with the induced Riemannian metric, and denote by $G(g)$ the space of unoriented geodesics of $\tilde{g}$ furnished with the compact-open topology; $G(g)$ is homeomorphic to the open Möbius band. (It is just $\left(S^{\infty} \times S^{\infty}-\right.$ Diag $) /\left\{\left(\theta_{1}, \theta_{2}\right) \sim\left(\theta_{2}, \theta_{1}\right)\right\}$ where $S^{\infty}$ is the circle at infinity.) A geodesic current of $(N, g)$ is a locally finite Borel measure on $G(g)$ invariant under the (isometric) action of the fundamental group $\pi_{1}(N)$. A geodesic current can also be interpreted as an invariant transverse measure on the geodesic foliation $\mathcal{F}$ of the projective tangent bundle $P T(N)$ (see [B]). We will let $C(N)$ represent the space of geodesic currents endowed with the weak* topology. The set $\mathcal{C}$ of conjugacy classes of the fundamental group $\pi_{1}(N)$ embeds in $C(N)$ as follows. Represent each prime conjugacy class (i.e., not an integer multiple of another class) $\langle\gamma\rangle$ by the unique closed geodesic, $\gamma$, on $N$ representing the class. The lifts of $\gamma$ to $\tilde{N}$ form a set of geodesics on $\tilde{N}$ which is invariant under the action of $\pi_{1}(N)$ and is discrete in the space $G(g)$. We associate $\langle\gamma\rangle$ with the geodesic current $j(\langle\gamma\rangle)$ that is the Dirac measure concentrated on the so-obtained set of geodesics of $\tilde{N}$. For an arbitrary conjugacy class of the fundamental group, the corresponding geodesic current is defined by homogeneity: $j\left(\left\langle\gamma^{p}\right\rangle\right)=|p| j(\langle\gamma\rangle)$. Geodesic currents can be added and multiplied by nonnegative reals to yield other geodesic currents.

The following, observed in [Bo], is a straightforward corollary to Sigmund's theorem [Si]:

Proposition 2.1. [Bo, Proposition 2] The space $C(S)$ is complete and the real multiples of the geodesic currents $j(\langle\gamma\rangle), \gamma \in \pi_{1}(S)$, coming from closed geodesics are dense in $C(S)$.

The canonical Liouville form $\lambda_{g}$ on the projective tangent bundle $P T(N)$ induces an invariant transverse measure on the geodesic foliation $\mathcal{F}$ of the projective tangent bundle $P T(N)$ which is generated by the 2 -form equal to half of the interior product of the Liouville form with the geodesic flow vector field. The resulting geodesic current is called the Liouville current of the surface $(N, g)$ and denoted by the same symbol $\lambda_{g}$ (see [Bo, Ot1]).

In [Bo] Bonahon constructed a continuous symmetric bilinear form $i$ : $C(S) \times C(S) \rightarrow \mathbb{R}^{+}$, called the intersection form. (On the dense set of the currents coming from closed geodesics $\gamma_{1}$ and $\gamma_{2}, i\left(\left\langle\gamma_{1}\right\rangle,\left\langle\gamma_{2}\right\rangle\right)$ is just the number of intersection points of $\gamma_{1}$ with $\gamma_{2}$.) Bonahon showed that $i$ enjoys the following properties relative to the Liouville current $\lambda_{g}$ :

Proposition 2.2. (a) For every conjugacy class $\langle\gamma\rangle$ of the fundamental group $\pi_{1}(N)$ we have $i\left(j(\langle\gamma\rangle), \lambda_{g}\right)=L_{g}(\langle\gamma\rangle)$.

(b) $i\left(\lambda_{g}, \lambda_{g}\right)=(\pi / 2) A(g)$.

In the above $L_{g}(\langle\gamma\rangle)$ represents the length of the closed geodesic in the class $\langle\gamma\rangle$. Property (a) was proved in [Bo, Proposition 14], [Ot1, Proposition 3]. Property (b) was proved in [Bo, Proof of Proposition 15]. 
The equality case of Theorem 1.1 relies on a recent positive Livschits type theorem of Lopes and Thieullen [L-T]. The consequence (special case) that we will use is:

Proposition 2.3. Let $A$ be a Hölder function on the unit tangent bundle of an n-dimensional negatively curved manifold whose integral along every closed geodesic is nonnegative. If the integral of $A$ over the unit tangent bundle is 0 then the integral of $A$ along every closed geodesic is identically 0.

Proof. To apply the result in [L-T] we note that the geodesic flow is Anosov, and that our assumption along with Sigmund's theorem [Si] implies that the integral of $A$ with respect to any flow invariant probability measure is nonnegative. Thus [L-T] gives us a Hölder function $V$ on the unit tangent bundle which is differentiable (with Hölder derivative $V^{\prime}$ ) in the direction of the flow such that $A(v) \geq V^{\prime}(v)$. Thus, since $0 \leq \int_{U N}\left(A(v)-V^{\prime}(v)\right) d \lambda_{g_{0}}=\int_{U N} A(v) d \lambda_{g_{0}}=0$, we see that $A(v)=V^{\prime}(v)$ and the result follows.

Proof of Theorem 1.1. We consider two metrics $g_{0}$ and $g_{1}$ of negative curvature on the compact surface $N$. The geodesic flows of $g_{0}$ and $g_{1}$ are orbit equivalent $[\mathrm{Gr} 2, \S 8]$. In particular, there exists a unique $\pi_{1}(N)$-equivariant homeomorphism between $G\left(g_{0}\right)$ and $G\left(g_{1}\right)$. Denote by $\lambda_{g_{1}}^{*}$ the pull-back of the Liouville current $\lambda_{g_{1}}$ of the metric $g_{1}$ under this homeomorphism. Then $\lambda_{g_{1}}^{*}$ is a geodesic current of the surface $\left(N, g_{0}\right)$. From the hypothesis of the theorem and Proposition 1.2(a) it follows that for all $\gamma \in \pi_{1}(N)$

$$
i\left(j(\langle\gamma\rangle), \lambda_{g_{0}}\right)=L_{g_{0}}(\langle\gamma\rangle) \leq L_{g_{1}}(\langle\gamma\rangle)=i\left(j(\langle\gamma\rangle), \lambda_{g_{1}}^{*}\right) .
$$

In view of Proposition 2.1 and the continuity of $i$

$$
i\left(\mu, \lambda_{g_{0}}\right) \leq i\left(\mu, \lambda_{g_{1}}^{*}\right)
$$

for all $\mu \in C(N)$. Using this fact twice (with the symmetry of $i$ ) and using Proposition 2.2(b) we get the inequality:

$$
(\pi / 2) A\left(g_{0}\right)=i\left(\lambda_{g_{0}}, \lambda_{g_{0}}\right) \leq i\left(\lambda_{g_{0}}, \lambda_{g_{1}}^{*}\right) \leq i\left(\lambda_{g_{1}}^{*}, \lambda_{g_{1}}^{*}\right)=(\pi / 2) A\left(g_{1}\right) .
$$

Now assume that equality holds. Let $\Phi^{t}$ be the geodesic flow of $g_{0}$ on the $g_{0}$-unit tangent bundle $U N$ and let $\Psi^{t}$ be the the geodesic flow of $g_{1}$ on the $g_{1}$ unit tangent bundle $U_{1} N$. Let $f$ be the orbit equivalence, i.e $f: U N \rightarrow U_{1} N$ is a homeomorphism, homotopic to the mapping induced by the identity mapping $N \rightarrow N$, such that the flow $f \circ \Phi^{t} \circ f^{-1}$ is a time change of $\Psi^{t}$. It means that for some $\alpha: U N \times \mathbb{R} \rightarrow \mathbb{R}$ we have

$$
f\left(\Phi^{t}(v)\right)=\Psi^{\alpha(v, t)}(f(v))
$$

for all $v \in U N$ and $t \in \mathbb{R}$. Moreover, by [KH, Chap. 19] we may assume that $f$ is Hölder continuous, and so is $\alpha$.

Now, observe that by the calculations in [Bo]

$$
i\left(\lambda_{g_{0}}, \lambda_{g_{1}}^{*}\right)=\frac{1}{4} \int_{U N} \alpha(v, 1) d \lambda_{g_{0}} .
$$


Another way to see this is to notice that for every closed $g_{0}$-geodesic $\gamma$

$$
i\left(\langle\gamma\rangle, \lambda_{g_{1}}^{*}\right)=\int_{0}^{L_{g_{0}}(\gamma)} \alpha(\dot{\gamma}(s), 1) d s,
$$

because the left-hand side equals $L_{g_{1}}(\gamma)$ by Proposition 2.2(a) while the righthand side equals (putting $v=\dot{\gamma}(0)$ and $L=L_{g_{0}}(\gamma)$ )

$$
\begin{gathered}
\int_{0}^{L} \alpha\left(\Phi^{s}(v), 1\right) d s=\int_{0}^{L}(\alpha(v, s+1)-\alpha(v, s)) d s \\
=\int_{0}^{1}(\alpha(v, L+s)-\alpha(v, s)) d s=\alpha\left(\Phi^{L} v, L\right)=\alpha(v, L)=L_{g_{1}}(\gamma) .
\end{gathered}
$$

Next, one should use Sigmund's theorem to approximate $\lambda_{g_{0}}$.

This also shows that under the assumptions of the theorem the function $A(v)=\alpha(v, 1)-1$ on $U N$ has nonnegative integrals with respect to all invariant measures on $U N$. Now, if $A\left(g_{0}\right)=A\left(g_{1}\right)$, it follows that $i\left(\lambda_{g_{0}}, \lambda_{g_{1}}^{*}\right)=$ $i\left(\lambda_{g_{0}}, \lambda_{g_{0}}\right)=\frac{\pi}{2} A\left(g_{0}\right)$. Therefore,

$$
\int_{U N} \alpha(v, 1) d \lambda_{g_{0}}-\int_{U N} 1 d \lambda_{g_{0}}=4 i\left(\lambda_{g_{0}}, \lambda_{g_{1}}^{*}\right)-2 \pi A\left(g_{0}\right)=0 .
$$

Thus $A(v)$ integrates to 0 and Proposition 2.3 says that the marked length spectra are the same. The result now follows from the marked length spectrum rigidity theorem for a negatively curved surface of [Cr2, Ot1]. (For marked length spectrum rigidity theorems for nonpositively curved surfaces see [C-F-F, Ba].)

Proof of Theorem 1.2. Let $N$ be compact without boundary and $g_{0}$ be a Riemannian metric of negative curvature on $N$ and $g_{1}=f^{2}(x) g_{0}$ be a pointwise conformal metric whose marked length spectrum is greater than or equal to that of $g_{0}$. We show:

$\operatorname{Vol}\left(g_{1}\right) \geq \operatorname{Vol}\left(g_{0}\right)$ with equality if and only if $f(x)=1$.

We do this in two steps by considering the quantity

$$
I=\int_{U N}|u|_{1} d_{0} u
$$

where $U N$ is the $g_{0}$-unit tangent bundle of $N,|u|_{1}$ is the length of the $g_{0}$-unit vector $u$ with respect to the metric $g_{1}$, and $d_{0} u$ represents the Liouville measure with respect to $g_{0}$. That is $|u|_{1}=f(x)$ where $x \in M$ is the base point of $u$.

Step 1 is just the Hölder inequality (where $\omega(n-1)$ represents the volume of the unit $n-1$ sphere):

$$
\begin{gathered}
I=\omega(n-1) \int_{N} f(x) d x \leq \omega(n-1)\left\{\int_{N} 1 d x\right\}^{\frac{n-1}{n}}\left\{\int_{N} f^{n}(x) d x\right\}^{\frac{1}{n}} \\
=\omega(n-1) \operatorname{Vol}\left(g_{0}\right)^{\frac{n-1}{n}} \operatorname{Vol}\left(g_{1}\right)^{\frac{1}{n}} .
\end{gathered}
$$

which says that $I \leq \omega(n-1) \operatorname{Vol}\left(g_{0}\right)^{\frac{n-1}{n}} \operatorname{Vol}\left(g_{1}\right)^{\frac{1}{n}}$ with equality holding if and only if $f$ is constant.

Step 2 shows that $I \geq \omega(n-1) \operatorname{Vol}\left(g_{0}\right)$ which when combined with step 1 yields the result. We let $f(u): U N \rightarrow R$ be the function induced by $f$ (i.e. 
$f(u)=f(x)$ where $x$ is the base point of $u$ ). So $I=\int_{U N} f(u) d_{0} u$. Using Sigmund's theorem [Si] there is a sequence, $\left\{\gamma_{i}\right\}$, of $g_{0}$-closed geodesics of lengths $L_{i}$ such that for any function $h: U N \rightarrow R$ we have:

$$
\frac{1}{\operatorname{Vol}(U N)} \int_{U N} h(u) d_{0} u=\lim _{i \rightarrow \infty} \frac{1}{L_{i}} \int_{0}^{L_{i}} h\left(\gamma_{i}^{\prime}(t)\right) d t .
$$

For every $g_{0}$-closed geodesic $\gamma, \int_{0}^{L_{i}} f\left(\gamma^{\prime}(t)\right) d t$ represents the length of $\gamma$ in the $g_{1}$ metric, which is longer than the shortest closed $g_{1}$-geodesic freely homotopic to $\gamma$, which in turn, by the condition on the marked length spectrum, is greater than the $g_{0}$-length of $\gamma$. Thus

$$
\frac{I}{\operatorname{Vol}(U N)}=\lim _{i \rightarrow \infty} \frac{1}{L_{i}} \int_{0}^{L_{i}} f\left(\gamma_{i}^{\prime}(t)\right) d t \geq 1 .
$$

\section{Manifolds with Boundary}

Let $(M, \partial M, g)$ be a compact SGM Riemannian manifold with boundary. There is a canonical measure $d \gamma$ on the space $\Gamma_{g}$ of nongrazing unit speed geodesics (which in the $S G M$ case run from a boundary point to a boundary point) such that for every integrable function $f$ on the unit tangent bundle we have:

$$
\int_{\Gamma_{g}} \int_{0}^{L(\gamma)} f\left(\gamma^{\prime}(t)\right) d t d \gamma=\int_{U M} f(u) d u,
$$

where $d u$ is the Liouville measure and $L(\gamma)$ is the length of $\gamma$. In particular, by plugging in $f=1$ and letting $\omega(n-1)$ represent the volume of the unit $(n-1)$-sphere,

$$
\omega(n-1) \operatorname{Vol}(M)=\operatorname{Vol}(U M)=\int_{\Gamma_{g}} L(\gamma) d \gamma .
$$

Given a codimension 1 submanifold $N$ of $M$ with normal vector $\nu$, the geodesics passing through $N$ can be parameterized by the unit vectors $V$ tangent to $M$ with base point on $N$. Santaló's formula [Sa1], [Sa2, Chap. 19] allows us to express $d \gamma$ on this subset by $d \gamma=|\langle\nu, v\rangle| d v$, where $d v$ is the measure which locally is the product of the Riemannian measure on $N$ (the base point) with the standard measure on a unit sphere. We will use this in two ways.

In the $S G M$ case we can parameterize the geodesic segments by their initial tangents $v \in U^{+} \partial M$ where $U^{+} \partial M$ represents the unit tangent vectors $v$ to $M$ whose base points are on $\partial M$ and which point "inward" (i.e. $\langle v, \nu\rangle \geq 0$, where $\nu$ is the inward normal). Santaló's formula allows us to express $d \gamma$ as $\langle\nu, v\rangle d v$. Another immediate application is Crofton's formula for geodesics which says that $C(n-1) \operatorname{Vol}(N)$, for a codimension one submanifold $N$, is just the total $d \gamma$-measure of the set of geodesics that intersect $N$ with each geodesic counted with multiplicity equal to the number of intersections with $N$. Here $C(n-1)$ is the integral over the unit sphere of $|\langle v, \nu\rangle|$. We will use this in 2-dimensions where $N$ is a curve to conclude that 4 times the length is measure of such geodesics counted with multiplicity.

Proof of Theorem 1.3. We let $M$ be an $n$-dimensional manifold with boundary which is endowed with two $S G M$ metrics: $g_{0}$ with volume $V_{0}=$ 
$\int_{M} d x_{0}$ and $g_{1}=f(x) g_{0}$ of volume $V_{1}=\int_{M} f^{n}(x) d x_{0}$. For $\gamma \in \Gamma_{g_{0}}$ and $i=1,2$, we let $L_{i}(\gamma)$ represent the length of $\gamma$ in the $g_{i}$ metric while $\mathcal{L}_{i}(\gamma)$ will represent the distance in $g_{i}$ between the endpoints of $\gamma$. Of course $L_{0}(\gamma)=\mathcal{L}_{0}(\gamma)$ while $L_{1}(\gamma) \geq \mathcal{L}_{1}(\gamma)$. Note that $L_{1}(\gamma)=\int_{0}^{L_{0}(\gamma)} f(\gamma(t)) d t$. In what follows $c_{1}=$ $\omega(n-1)^{-1}, d_{0} x$ is the $g_{0}$ Riemannian volume form on $M$, and $d_{0} \gamma$ is the $g_{0}$ measure on $\Gamma_{g_{0}}$.

By Hölder's inequality:

$$
\int_{M} f^{2}(x) d_{0} x \leq\left\{\int_{M} f^{n}(x) d_{0} x\right\}^{\frac{2}{n}}\left\{\int_{M} d_{0} x\right\}^{\frac{n-2}{n}}=\operatorname{Vol}\left(g_{1}\right)^{\frac{2}{n}} \operatorname{Vol}\left(g_{0}\right)^{\frac{n-2}{n}} .
$$

Using (3.1) (twice) we see:

$$
\begin{gathered}
\int_{M} f(x) d_{0} x=c_{1} \int_{\gamma \in \Gamma_{g_{0}}} \int_{0}^{L_{0}(\gamma)} f(\gamma(t)) d t d_{0} \gamma=c_{1} \int_{\gamma \in \Gamma_{0}} L_{1}(\gamma) d_{0} \gamma \\
=c_{1} \int_{\gamma \in \Gamma_{g_{0}}} L_{0}(\gamma) d_{0} \gamma+c_{1} \int_{\gamma \in \Gamma_{g_{0}}}\left(L_{1}(\gamma)-L_{0}(\gamma)\right) d_{0} \gamma \\
=\int_{M} d_{0} x+c_{1} \int_{\gamma \in \Gamma_{g_{0}}}\left(L_{1}(\gamma)-L_{0}(\gamma)\right) d_{0} \gamma
\end{gathered}
$$

and hence

$$
\begin{gathered}
2 \int_{M}(1-f(x)) d_{0} x=2 c_{1} \int_{\gamma \in \Gamma_{g_{0}}}\left(L_{0}(\gamma)-L_{1}(\gamma)\right) d_{0} \gamma \\
\leq 2 c_{1} \int_{\gamma \in \Gamma_{g_{0}}}\left(\mathcal{L}_{0}(\gamma)-\mathcal{L}_{1}(\gamma)\right) d \gamma_{0}
\end{gathered}
$$

Thus since

$$
\int_{M}(1-f(x))^{2} d_{0} x=\int_{M} f(x)^{2} d_{0} x+2 \int_{M}(1-f(x)) d_{0} x-\operatorname{Vol}\left(g_{0}\right)
$$

using (3.3) and (3.4) we arrive at the stability estimate:

$$
\begin{aligned}
\int_{M}(1-f(x))^{2} d_{0} x \leq & \left(\operatorname{Vol}\left(g_{1}\right)^{\frac{2}{n}}-\operatorname{Vol}\left(g_{0}\right)^{\frac{2}{n}}\right) \operatorname{Vol}\left(g_{0}\right)^{\frac{n-2}{n}} \\
& +2 c_{1} \int_{\gamma \in \Gamma g_{0}} \mathcal{L}_{0}(\gamma)-\mathcal{L}_{1}(\gamma) d_{0} \gamma .
\end{aligned}
$$

In particular, if $\mathcal{L}_{1} \geq \mathcal{L}_{0}$ (i.e $\left.d_{g_{1}} \geq d_{g_{0}}\right)$ then $V_{g_{1}} \geq V\left(g_{0}\right)$, yielding the inequality of the theorem. Further if the volumes are equal then the right hand side is nonpositive and hence $f \equiv 1$. Note that if we assume $\mathcal{L}_{0}=\mathcal{L}_{1}$ then by (3.2) we also have $V\left(g_{0}\right)=V\left(g_{1}\right)$ and we get the boundary rigidity result that $f \equiv 1$.

We now consider the case of compact $S G M$ surfaces $(M, \partial M, g)$ with boundary. We will make an intersection type argument. We will call $(x, y,[\alpha]) \in \mathcal{A}$ (see the introduction for a definition) $g$-regular if there is a nongrazing geodesic segment from $x$ to $y$ and this segment is not tangent to the boundary at either endpoint. The $g$-regular set is of full measure with respect to $\mu_{g}$. The main tool in our study is: 
Proposition 3.1. If $g_{0}$ and $g_{1}$ are SGM Riemannian metrics on a compact surface $(M, \partial M)$ with boundary $\partial M$ then

$$
\int_{\mathcal{A}} L_{g_{0}}(x, y,[\alpha]) f_{g_{1}}(x, y,[\alpha]) d x d y=\int_{\mathcal{A}} L_{g_{1}}(x, y,[\alpha]) f_{g_{0}}(x, y,[\alpha]) d x d y .
$$

Proof. We consider $\mathcal{A} \times \mathcal{A}$ as the measure space with the product measure $\mu_{g_{0}} \times$ $\mu_{g_{1}}$ and consider the geometric intersection number $i\left(\left(x_{0}, y_{0},\left[\alpha_{0}\right]\right),\left(x_{1}, y_{1},\left[\alpha_{1}\right]\right)\right)$ which is the minimum number of intersection points of two curves, one representing $\left[\alpha_{0}\right]$ and one representing $\left[\alpha_{1}\right]$. (Here if for two curves $\sigma$ and $\tau$ there are parameter values $s_{1}, s_{2}$ and $t$ such that $\sigma\left(s_{1}\right)=\tau(t)$ and $\sigma\left(s_{2}\right)=\tau(t)$ then this counts as two intersection points.) We claim that both sides of the equation in the statement of the proposition represent

$$
\frac{1}{4} \int_{\mathcal{A} \times \mathcal{A}} i\left(\left(x_{0}, y_{0},\left[\alpha_{0}\right]\right),\left(x_{1}, y_{1},\left[\alpha_{1}\right]\right)\right) d \mu_{g_{0}}\left(x_{0}, y_{0},\left[\alpha_{0}\right]\right) \times d \mu_{g_{1}}\left(x_{1}, y_{1},\left[\alpha_{1}\right]\right) .
$$

We will show this for the right hand side. The other side is similar. By Fubini's theorem we need only show that for each fixed $g_{0}$-regular $\left(x_{0}, y_{0},\left[\alpha_{0}\right]\right)$ :

$$
\frac{1}{4} \int_{\mathcal{A}} i\left(\left(x_{0}, y_{0},\left[\alpha_{0}\right]\right),\left(x_{1}, y_{1},\left[\alpha_{1}\right]\right)\right) d \mu_{g_{1}}\left(x_{1}, y_{1},\left[\alpha_{1}\right]\right)=L_{g_{1}}\left(x_{0}, y_{0},\left[\alpha_{0}\right]\right) .
$$

In this integral we can ignore $\left\{\left(x_{1}, y_{1},\left[\alpha_{1}\right]\right) \mid\left\{x_{0}, y_{0}\right\} \cap\left\{x_{1}, y_{1}\right\} \neq \emptyset\right\}$ since it has measure 0 . Let $\tau$ be a shortest $g_{1}$ path from $x_{0}$ to $y_{0}$ in $\left[\alpha_{0}\right]$. We will show that if $\left(x_{1}, y_{1},\left[\alpha_{1}\right]\right)$ is $g_{1}$-regular and $\gamma$ is the $g_{1}$-geodesic segment from $x_{1}$ to $y_{1}$ in $\left[\alpha_{1}\right]$ then $\gamma$ will intersect $\tau$ in precisely $i\left(\left(x_{0}, y_{0},\left[\alpha_{0}\right]\right),\left(x_{1}, y_{1},\left[\alpha_{1}\right]\right)\right)$ points. The result will then follow from the version of Crofton's formula we discussed above. We note that, since $\left(x_{1}, y_{1},\left[\alpha_{1}\right]\right)$ is $g_{1}$-regular and since $\gamma$ and $\tau$ do not share endpoints, $\gamma$ will intersect $\tau$ at finitely many interior points of $M$. To count the number of intersection points of $\gamma$ and $\tau$ we can instead lift $\tau$ to a curve $\tilde{\tau}$ in the universal cover $\tilde{M}$ and count the number, $n$, of lifts, $\tilde{\gamma}_{i}$, of $\gamma$ whose intersection with $\tilde{\tau}$ is nonempty. The homotopy minimizing properties of $\tau$ and $\gamma$ imply that $\tilde{\tau}$ and $\tilde{\gamma}_{i}$ in fact minimize distance between endpoints and hence they intersect at at most one point. So $\tau$ and $\gamma$ intersect in exactly $n$ points. Now if $\tau^{\prime}$ and $\gamma^{\prime}$ are curves homotopic to $\tau$ and $\gamma$ relative to the endpoints then the corresponding lifts (i.e. lifts having the same endpoints) $\tilde{\tau}^{\prime}$ and $\tilde{\gamma}^{\prime}$ will have oriented intersection number 1 and hence must intersect. So $\tau^{\prime}$ and $\gamma^{\prime}$ intersect at least $n$ times. Thus $n$ must be equal to $i\left(\left(x_{0}, y_{0},\left[\alpha_{0}\right]\right),\left(x_{1}, y_{1},\left[\alpha_{1}\right]\right)\right)$.

The proof of Theorem 1.4 follows immediately from this proposition and the area formula $\int_{\mathcal{A}} L_{g}(x, y,[\alpha]) \mu_{g}=2 \pi A(g)$.

Proof of Corollary 1.5. From Theorem 1.4 we need only see the inequality $L_{g_{1}}(x, y,[\alpha]) \geq L_{g_{0}}(x, y,[\alpha])$ whenever either $f_{g_{1}}(x, y,[\alpha])$ or $f_{g_{0}}(x, y,[\alpha])$ is not zero. If $f_{g_{0}}(x, y,[\alpha])$ is not zero then the general assumption $d_{g_{1}} \geq d_{g_{0}}$ gives the result. If $f_{g_{1}}(x, y,[\alpha])$ is not zero then the other assumption is exactly the condition $L_{g_{1}}(x, y,[\alpha]) \geq L_{g_{0}}(x, y,[\alpha]$.

Equality holds above if and only if for all $(x, y,[\alpha])$ that are either $g_{0}$-regular or $g_{1}$-regular we have $L_{g_{1}}(x, y,[\alpha])=L_{g_{0}}(x, y,[\alpha])$. Other pairs $x, y$ of boundary points in the equality case of the corollary that are not covered by the above are limits of ones above. 


\section{References}

[Ba] V. Bangert, Geodesic rays, Busemann functions and monotone twist maps, Calc. Var. Part. Diff. Eq. 2 (1994), no. 1, 49-63.

[B-C-G1] G. Besson, G. Courtois, S. Gallot, Entropies et rigidités des espaces localement symétriques de courbure strictement négative, Geom. Funct. Anal. 5 (1995), no. 5, 731-799.

[B-C-G2] G. Besson, G. Courtois, S. Gallot, Minimal entropy and Mostow's rigidity theorems, Ergodic Theory Dynam. Systems 16 (1996), no. 4, 623-649.

[Bo] F. Bonahon, The geometry of Teichmüller space via geodesic currents, Inven. Math. 33 (1991), 445-464.

[Cr1] C. Croke, Rigidity and the distance between boundary points, J. Diff. Geom. 92 (1988), 139-163.

[Cr2] C. Croke, Rigidity for surfaces of non-positive curvature, Comm. Math. Helv. 65 (1990), no. 1, 150-169.

[Cr3] C. Croke, Rigidity Theorems in Riemannian geometry, (preprint - to appear in "Geometry, Inverse Problems and Control of PDE" based on the conference at IMA, July 2001).

[C-D-S] C. Croke, N. Dairbekov, and V. Sharafutdinov, Local boundary rigidity of a compact Riemannian manifold with curvature bounded above, Trans. Amer. Math. Soc. 352 (2000), no. 9, 3937-3956.

[C-F-F] C. Croke, A. Fathi, and J. Feldman, The marked length-spectrum of a surface of nonpositive curvature, Topology 31 (1992), no. 4, 847-855.

[Gr1] M. Gromov, Filling Riemannian manifolds, J. Diff. Geom. 18 (1983), $1-147$.

[Gr2] M. Gromov, Hyperbolic Groups, in Essays in Group Theory, MSRI pub. (1987), vol. 8, 75-263.

[Iv] S. Ivanov, On two-dimensional minimal fillings, St. Petersbg. Math. J. 13 (2002), no. 1, 17-25.

[KH] A. Katok and B. Hasselblat, Introduction to the Modern Theory of Dynamical Systems. Encyclopedia of Mathematics and its Applications, Vol. 54. Cambridge University Press, Cambridge, 1995.

[L-T] A.O. Lopes and Ph. Thieullen, Sub-actions for Anosov flows, (preprint).

[Sa1] L. A. Santaló, Measure of sets of geodesics in a Riemannian space and applications to integral formulas in elliptic and hyperbolic spaces, Summa Brasil. Math. 3, (1952). 1-11.

[Sa2] L. A. Santaló, Integral Geometry and Geometric Probability. With a Foreword by Mark Kac. Encyclopedia of Mathematics and its Applications, Vol. 1. Addison-Wesley Publishing Co., Reading, Mass.London-Amsterdam, 1976. 
[Si] K. Sigmund, On the space of invariant measures for hyperbolic flows, Ann. of Math. 131(1990), 151-162.

[Ot1] J.-P. Otal, Le spectre marqué des longueurs des surfaces à courbure négative, Amer. J. Math. 94(1972), 31-37.

[Ot2] J.-P. Otal, Sur les longueurs des géodésiques d'une métrique à courbure négative dans le disque, Comment. Math. Helv. 65 (1990), no. 2, $334-347$.

Christopher B Croke:

Department of Mathematics

University of Pennsylvania

Philadelphia, PA 19104-6395

ccroke@math.upenn.edu

Nurlan S. Dairbekov:

Sobolev Institute of Mathematics

Novosibirsk, Russia

dair@math.nsc.ru 